\title{
Extracting Equation of State Parameters from Black Hole-Neutron Star Mergers
}

\author{
Benjamin D. Lackey ${ }^{1}$, Koutarou Kyutoku ${ }^{2}$, Masaru Shibata ${ }^{2}$, Patrick R. Brady ${ }^{1}$, John L. Friedman ${ }^{1}$ \\ ${ }^{1}$ Department of Physics, University of Wisconsin-Milwaukee, Milwaukee, WI, USA \\ ${ }^{2}$ Yukawa Institute for Theoretical Physics, Kyoto University, Kyoto, Japan
}

\begin{abstract}
The late inspiral, merger, and ringdown of a black hole-neutron star (BHNS) system can provide information about the neutron-star equation of state (EOS). Candidate EOSs can be approximated by a parametrized piecewise-polytropic EOS, and we report results from a large set of BHNS inspiral simulations that systematically vary two parameters. To within the accuracy of the simulations, we find that a single physical parameter $\Lambda$, describing its deformability, can be extracted from the late inspiral, merger, and ringdown waveform. This parameter is related to the radius, mass, and $\ell=2$ Love number, $k_{2}$, of the neutron star by $\Lambda=2 k_{2} R^{5} / 3 M_{\mathrm{NS}}^{5}$, and it is the same parameter that determines the departure from point-particle dynamics during the early inspiral. Using various configurations of a single Advanced LIGO detector, we find that $\Lambda^{1 / 5}$ or equivalently $R$ can be extracted to $10-40 \%$ accuracy from single events for mass ratios of $Q=2$ and 3 at a distance of $100 \mathrm{Mpc}$, while with the proposed Einstein Telescope, EOS parameters can be extracted to accuracy an order of magnitude better.
\end{abstract}

\section{Introduction}

Construction of the second-generation Advanced LIGO (aLIGO) detectors is underway, and will soon begin for Advanced VIRGO and LCGT, making it likely that gravitational waveforms from compact binaries will be observed in this decade. Plans are also in development for the third generation Einstein Telescope (ET) detector with an order-of-magnitude increase in sensitivity over aLIGO. Population synthesis models predict that with a single aLIGO detector black hole-neutron star (BHNS) systems be observed with a signal-to-noise ratio (SNR) of 8 , at an event rate between 0.2 and 300 events per year at the same SNR and with a most likely value of 10 events per year for a canonical $1.4 M_{\odot}-10 M_{\odot}$ system [1]. The expected mass ratios $Q=M_{\mathrm{BH}} / M_{\mathrm{NS}}$ of BHNS systems are also highly uncertain and may range from just under 3 to more than 20 [2, 3].

A major goal of the gravitational-wave $(\mathrm{GW})$ program is to extract from observed waveforms the physical characteristics of their sources and, in particular, to use the waveforms of inspraling and merging binary neutron star (BNS) and BHNS systems to constrain the uncertain EOS of neutron-star matter. During inspiral the tidal interaction between the two stars leads to a small drift in the phase of the gravitational waveform relative to a point particle system. Specifically the tidal field $\mathcal{E}_{i j}$ of one star will induce a quadrupole moment $Q_{i j}$ in the other star given by $Q_{i j}=-\lambda \mathcal{E}_{i j}$ where $\lambda[24]$ is an EOS dependent quantity that describes how easily the star is distorted. A method for determining $\lambda$ for relativistic stars was found by Hinderer [4]; its effect on the waveform was calculated to Newtonian order (with the relativistic value of $\lambda$ ) by Flanagan and Hinderer [5] and to first post-Newtonian (PN) order by Vines, Flanagan, and Hinderer [6, 7]. This tidal description has also been extended to higher order multipoles $[8,9]$.

Numerical BHNS simulations have been done to examine the dependence of the waveform on mass ratio, BH spin, NS mass, and the neutron-star EOS. However, an analysis of the detectability of EOS information with GW detectors using these simulations has not yet been done, and the present paper presents the first results of this kind. EOS information from tidal interactions is present in the inspiral waveform. For BHNS systems, however, the stronger signal is likely to arise from a sharp drop in the GW amplitude arising from tidal disruption prior to merger or, when there is negligible disruption, from the cutoff frequency at merger [10].

We find from simulations of the last few orbits, merger, and ringdown of BHNS systems with varying

[1] We thank Alessandro Nagar for significant help with understanding the EOB formalism, Yi Pan and Alessandra Buonanno for providing EOB waveforms with spinning black holes, Jocelyn Read for providing routines used in the data analysis, and Jolien Creighton for generating narrowband noise curves. This work was supported by NSF Grants PHY-1001515 and PHY-0923409, by Grant-in-Aid for Scientific Research (21340051), by Grant-in-Aid for Scientific Research on Innovative Area (20105004) of Japanese MEXT, and by a Grant-in-Aid of JSPS. BL would also like to acknowledge support from a UWM Graduate School Fellowship and the Wisconsin Space Grant Consortium. KK is also supported by the Grant-in-Aid for the Global COE Program "The Next Generation of Physics, Spun from Universality and Emergence" of Japanese MEXT. 
EOS that, to within numerical accuracy, the EOS parameter extracted from the waveform is the same tidal parameter $\Lambda$ that determines the departure from point particle behavior during inspiral; here $\Lambda$ is a dimensionless version of the tidal parameter:

$$
\Lambda:=G \lambda\left(\frac{c^{2}}{G M_{\mathrm{NS}}}\right)^{5}=\frac{2}{3} k_{2}\left(\frac{c^{2} R}{G M_{\mathrm{NS}}}\right)^{5}
$$

where $k_{2}$ is the quadrupole Love number.

Conventions: Unless otherwise stated we set $G=c=1$.

\section{Parametrized EOS}

To understand the dependence of the BHNS waveform on the EOS we systematically vary the free parameters of a parametrized EOS and then simulate a BHNS inspiral for each set of parameters. We choose the piecewise polytropic EOS introduced in Ref [11]. Within each density interval $\rho_{i-1}<\rho<\rho_{i}$, the pressure $p$ is given in terms of the rest mass density $\rho$ by

$$
p(\rho)=K_{i} \rho^{\Gamma_{i}}
$$

where the adiabatic index $\Gamma_{i}$ is constant in each interval, and the pressure constant $K_{i}$ is chosen so that the EOS is continuous at the boundaries $\rho_{i}$ between adjacent segments of the EOS. The energy density $\epsilon$ is found using the first law of thermodynamics,

$$
d \frac{\epsilon}{\rho}=-p d \frac{1}{\rho}
$$

Ref. [11] uses a fixed low density EOS for the NS crust. The parametrized high density EOS is then joined onto the low density EOS at a density $\rho_{0}$ that depends on the values of the high-density EOS parameters. The high-density EOS consists of a three-piece polytrope with fixed dividing densities $\rho_{1}=10^{14.7} \mathrm{~g} / \mathrm{cm}^{3}$ and $\rho_{2}=10^{15} \mathrm{~g} / \mathrm{cm}^{3}$ between the three polytropes. The resulting EOS has four free parameters. The first parameter, the pressure $p_{1}$ at the first dividing density $\rho_{1}$, is closely related to the radius of a $1.4 M_{\odot} \mathrm{NS}$ [12]. The other three parameters are the adiabatic indices $\left\{\Gamma_{1}, \Gamma_{2}, \Gamma_{3}\right\}$ for the three density intervals. This parametrization accurately fits a wide range of theoretical EOS and reproduces the corresponding NS properties such as radius, moment of inertia, and maximum mass to a few percent [11].

Following previous work on BNS [13] and BHNS simulations [14, 15] we use a simplified two-parameter version of the piecewise-polytrope parametrization and uniformly vary each of these parameters. For our two parameters we use the pressure $p_{1}$ as well as a single fixed adiabatic index $\Gamma=\Gamma_{1}=\Gamma_{2}=\Gamma_{3}$ for the core. The crust EOS is given by a single polytrope with the constants $K_{0}=3.5966 \times 10^{13}$ in cgs units and $\Gamma_{0}=1.3569$ so that the pressure at $10^{13} \mathrm{~g} / \mathrm{cm}^{3}$ is $1.5689 \times 10^{31} \mathrm{dyne} / \mathrm{cm}^{2}$. (For most values of $p_{1}, \Gamma_{1}$, and $\Gamma_{2}$, the central density of a $1.4 M_{\odot}$ star is below or just above $\rho_{2}$, so the parameter $\Gamma_{3}$ is irrelevant anyway for BNS before merger and BHNS for all times.)

We list in Table I the 21 EOS used in the simulations along with some of the NS properties. In addition, we plot the EOS as points in parameter space in Fig. 1 along with contours of constant radius, tidal deformability $\Lambda$, and maximum NS mass. The $1.93 M_{\odot}$ maximum mass contour corresponds to the recently observed pulsar with a mass of $1.97 \pm 0.04 M_{\odot}$ measured using the Shapiro delay [16]. In this two-parameter cross section of the full four-parameter EOS space, parameters below this curve are ruled out.

\section{Description of waveforms}

Using the 21 EOS described in Table I, we have performed 30 BHNS inspiral and merger simulations with different mass ratios $Q=M_{\mathrm{BH}} / M_{\mathrm{NS}}$ and neutron star masses $M_{\mathrm{NS}}$. A complete list of these simulations is given in Table II. For the mass ratio $Q=2$ and NS mass $M_{\mathrm{NS}}=1.35 M_{\odot}$, we performed a simulation for each of the 21 EOS. In addition, we performed simulations of a smaller NS mass $\left(Q=2, M_{\mathrm{NS}}=1.20 M_{\odot}\right)$ 
TABLE I: Neutron star properties for the 21 EOS used in the simulations. The original EOS names [13-15] are also listed. $p_{1}$ is given in units of dyne $/ \mathrm{cm}^{2}$, maximum mass is in $M_{\odot}$, and neutron star radius $R$ is in $\mathrm{km} . R, k_{2}$, and $\Lambda$ are given for the two masses used: $\{1.20,1.35\} M_{\odot}$.

\begin{tabular}{|c|c|c|c|c|c|c|c|c|c|}
\hline EOS & $\log p_{1}$ & $\Gamma$ & $M_{\max }$ & $R_{1.20}$ & $k_{2,1.20}$ & $\Lambda_{1.20}$ & $R_{1.35}$ & $k_{2,1.35}$ & $\Lambda_{1.35}$ \\
\hline Г2.4 Bss & 34.3 & 2.4 & 566 & .66 & 0.0765 & 401 & 10.27 & 0.0585 & - \\
\hline $2.7 \mathrm{Bs}$ & & 2.7 & 99 & & & 528 & & & \\
\hline $0 \mathrm{~B}$ & & 3.0 & 2.002 & & & 614 & & & \\
\hline & .3 & 3.3 & 2.181 & & & 677 & & & 33 \\
\hline$\Gamma 2.4$ HBss & 4.4 & 2.4 & & & & 755 & & & 01 \\
\hline Г2.7 HBs & 34.4 & 2.7 & 1.925 & 67 & & 828 & 57 & 55 & 75 \\
\hline $3.0 \mathrm{HB}$ & 4.4 & 3.0 & 2.122 & .60 & & 872 & & & 42 \\
\hline & 4.4 & 3.3 & & & & 903 & & & 45 \\
\hline & & 2.4 & & & & 1353 & & & 82 \\
\hline & & 2.7 & & & & 1271 & & & 59 \\
\hline $3.0 \mathrm{~F}$ & 4.5 & 3.0 & 2.249 & 12.25 & 55 & 1225 & 12 & 29 & 60 \\
\hline & 45 & 3.3 & 2.413 & 12.08 & & 1196 & & 85 & 61 \\
\hline & & 2.4 & & & & 2340 & & & 106 \\
\hline & & 2.7 & & & & 1920 & & & 932 \\
\hline & & 3.0 & & & & 1704 & & & 86 \\
\hline & & 3.3 & & 12 & & 1575 & & & 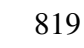 \\
\hline 4 & 4.7 & 2.4 & 2.180 & 15.35 & 0 & 3941 & 15 & 83 & 1860 \\
\hline & & 2.7 & & & & 2859 & & 144 & 1423 \\
\hline p.7Г3.0 1.5 & 34.7 & 3.0 & 2.525 & 13.62 & & 2351 & 13.69 & 0.1189 & 121 \\
\hline & & 3.3 & & & & 2062 & 13 & 0.1223 & 108 \\
\hline p. $9 Г 3.02 \mathrm{H}$ & 34.9 & 3.0 & 2.834 & 15.12 & 0.1453 & 4382 & 15.22 & 0.1342 & 232 \\
\hline
\end{tabular}

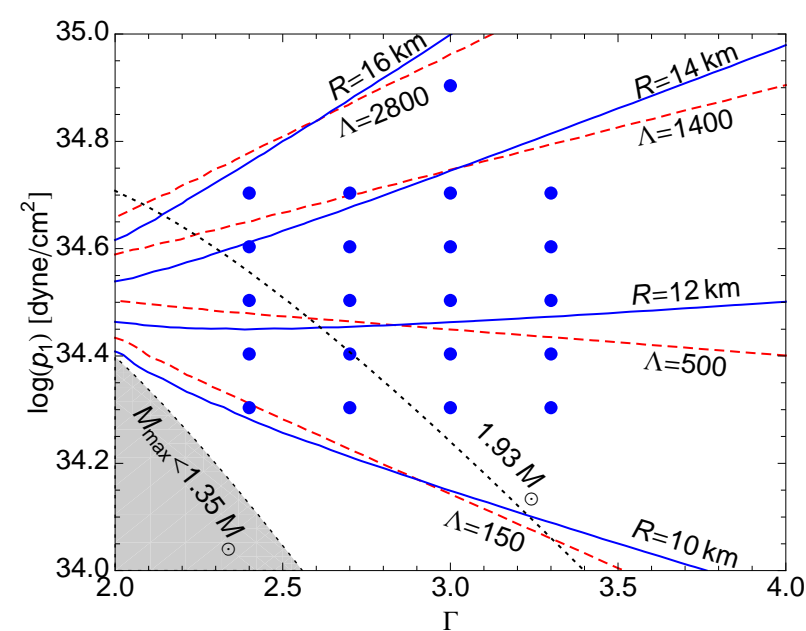

FIG. 1: The 21 EOS used in the simulations are represented by blue points in the parameter space. For a NS of mass $1.35 M_{\odot}$, contours of constant radius are solid blue and contours of constant tidal deformability $\Lambda$ are dashed red. Also shown are dotted contours of maximum NS mass. The shaded region does not allow a $1.35 M_{\odot}$ NS.

and a larger mass ratio $\left(Q=3, M_{\mathrm{NS}}=1.35 M_{\odot}\right)$, in which we only varied the pressure $p_{1}$ over the range $34.3 \leq \log \left(p_{1} /\left(\right.\right.$ dyne $\left.\left.\mathrm{cm}^{-2}\right)\right) \leq 34.9$ while holding the core adiabatic index fixed at $\Gamma=3.0$. 
TABLE II: Data for the 30 BHNS simulations. NS mass is in units of $M_{\odot}$, and $\Omega_{0} M$ is the angular velocity used in the initial data where $M=M_{\mathrm{BH}}+M_{\mathrm{NS}}$.

\begin{tabular}{cccc|cccc}
\hline \hline$Q$ & $M_{\mathrm{NS}}$ & EOS & $\Omega_{0} M$ & $Q$ & $M_{\mathrm{NS}}$ & EOS & $\Omega_{0} M$ \\
\hline 2 & 1.35 & p.3Г2.4 & 0.028 & 2 & 1.35 & p.6Г3.3 & 0.025 \\
2 & 1.35 & p.3Г2.7 & 0.028 & 2 & 1.35 & p.7Г2.4 & 0.025 \\
2 & 1.35 & p.3Г3.0 & 0.028 & 2 & 1.35 & p.7Г2.7 & 0.025 \\
2 & 1.35 & p.3Г3.3 & 0.025 & 2 & 1.35 & p.7Г3.0 & 0.028 \\
2 & 1.35 & p.4Г2.4 & 0.028 & 2 & 1.35 & p.7Г3.3 & 0.025 \\
2 & 1.35 & p.4Г2.7 & 0.028 & 2 & 1.35 & p.9Г3.0 & 0.025 \\
\cline { 3 - 7 } 2 & 1.35 & p.4Г3.0 & 0.028 & 2 & 1.20 & p.3Г3.0 & 0.028 \\
2 & 1.35 & p.4Г3.3 & 0.025 & 2 & 1.20 & p.4Г3.0 & 0.028 \\
2 & 1.35 & p.5Г2.4 & 0.025 & 2 & 1.20 & p.5Г3.0 & 0.028 \\
2 & 1.35 & p.5Г2.7 & 0.025 & 2 & 1.20 & p.9Г3.0 & 0.022 \\
\cline { 3 - 7 } 2 & 1.35 & p.5Г3.0 & 0.028 & 3 & 1.35 & p.3Г3.0 & 0.030 \\
2 & 1.35 & p.5Г3.3 & 0.025 & 3 & 1.35 & p.4Г3.0 & 0.030 \\
2 & 1.35 & p.6Г2.4 & 0.025 & 3 & 1.35 & p.5Г3.0 & 0.030 \\
2 & 1.35 & p.6Г2.7 & 0.025 & 3 & 1.35 & p.7Г3.0 & 0.030 \\
2 & 1.35 & p.6Г3.0 & 0.025 & 3 & 1.35 & p.9Г3.0 & 0.028 \\
\hline \hline
\end{tabular}

Two of the gravitational waveforms are shown in Fig. 2 below. The waveforms are compared with effective one body (EOB) binary black hole (BBH) waveforms of the same mass ratio and NS mass which are also shown. The most significant differences begin just before the merger of the black hole and neutron star. For neutron stars with a small radius, the black hole does not significantly distort the neutron star which crosses the event horizon intact. As a result, the merger and ringdown of these waveforms are very similar to the BBH waveform. However, a larger NS may be completely tidally disrupted just before merger resulting in a supressed merger and ringdown waveform. Disruption suppresses the ringdown for two reasons related to the spreading of the matter: The ringdown is primarily a superposition of nonaxisymmetric quasinormal modes, dominated by the $l=m=2$ mode, while the disrupted matter is roughly axisymmetric as it accretes onto the black hole; and the accretion timescale of the spread-out matter is long compared to the periods of the dominant modes.

The monotonic dependence of the waveform on $\Lambda$ can be seen in its Fourier transform $\tilde{h}$, shown in Fig. 3. The predicted EOS dependent frequency cutoff in the waveform [10] is clearly shown in the amplitude. Neutron stars that are more easily disrupted (larger $\Lambda$ ) result in an earlier and lower frequency drop in their waveform amplitude than NS with smaller $\Lambda$.

\section{Hybrid Waveform Construction}

Since our numerical simulations typically begin $\sim 5$ orbits before merger, it is necessary to join the numerical waveforms to analytic waveforms representing the earlier inspiral. There is a substantial literature comparing analytic and phenomenological waveforms with numerical waveforms extracted from simulations of BBH coalescence. For unequal mass systems, the EOB formalism (see Ref. [17] for a review) has proven to be a powerful tool to generate analytic waveforms that agree with numerical simulations.

We use a method similar to that described by Read et al. [13] to join each of the numerical BHNS waveforms to a reference EOB waveform, generating a hybrid EOB-numerical waveform. Denote a complex numerical waveform by $h_{\mathrm{NR}}(t)=h_{+}^{\mathrm{NR}}(t)-i h_{\times}^{\mathrm{NR}}(t)$ and an EOB waveform with the same $Q$ and $M_{\mathrm{NS}}$ by $h_{\mathrm{EOB}}(t)=h_{+}^{\mathrm{EOB}}(t)-i h_{\times}^{\mathrm{EOB}}(t)$. A constant time-shift $\tau$ and phase-shift $\Phi$ can be applied to the EOB waveform to match it to a section of the numerical waveform by rewriting it as $h_{\mathrm{EOB}}(t-\tau) e^{-i \Phi}$. We hold the numerical 

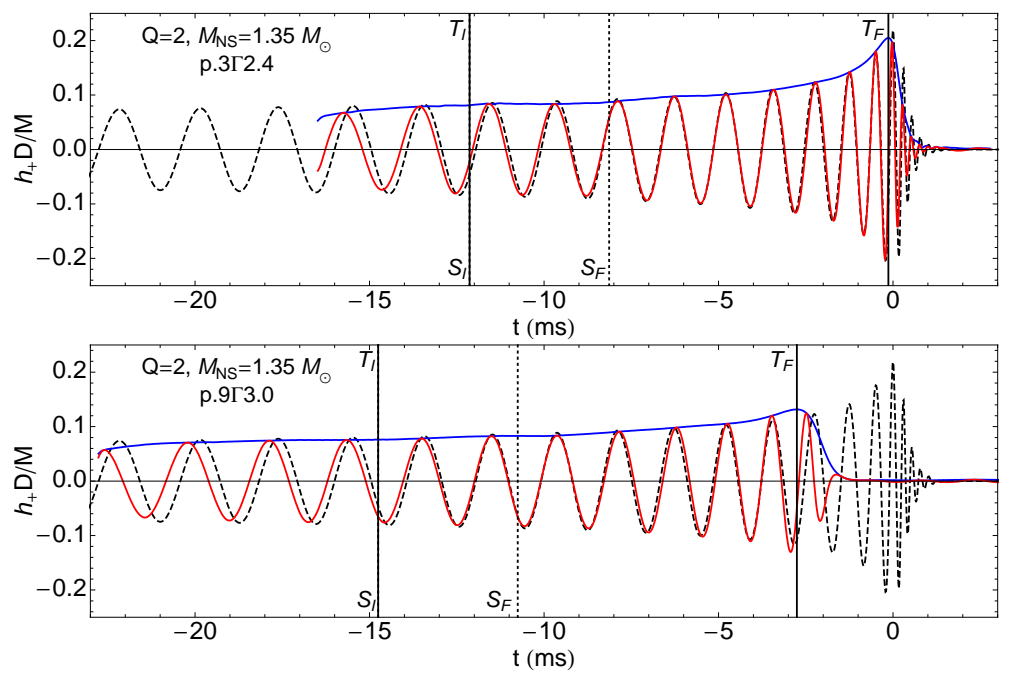

FIG. 2: $h_{+}$and $|h|=\left|h_{+}-i h_{\times}\right|$for BHNS waveforms for $\left(Q=2, M_{\mathrm{NS}}=1.35 M_{\odot}\right)$ with two different EOS are represented by solid red and blue curves respectively. The softest EOS p.3Г2.4 is on top and the stiffest EOS p.9Г3.0 is on bottom. An EOB BBH waveform (dashed) with the same values of $Q$ and $M_{\mathrm{NS}}$ is matched to each numerical waveform within the matching window $T_{I}<t<T_{F}$ bounded by solid vertical lines. A hybrid EOB BBH-Numerical BHNS waveform is generated by splicing the waveforms together within a splicing window $S_{I}<t<S_{F}$ bounded by dotted vertical lines. The matching window is $12 \mathrm{~ms}$ long and ends at the numerical merger time $t_{M}^{\mathrm{NR}}$ (time when the numerical waveform reaches its maximum amplitude), while the splicing window is $4 \mathrm{~ms}$ long and begins at the start of the matching window $\left(S_{I}=T_{I}\right)$.
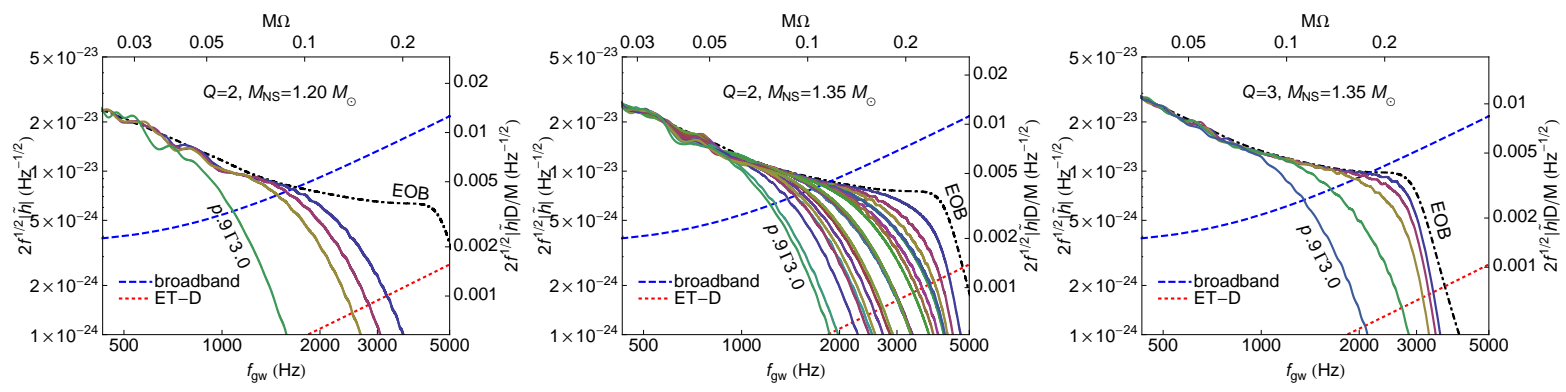

FIG. 3: Weighted Fourier transform $2 f^{1 / 2}|\tilde{h}(f)|$ of numerical waveforms where $\tilde{h}=\frac{1}{2}\left(\tilde{h}_{+}+\tilde{h}_{\times}\right)$. Dot-dashed curves are EOB waveforms with the same $Q$ and $M_{\mathrm{NS}}$. The left axis is scaled to a distance of $100 \mathrm{Mpc}$, and the noise $S_{n}^{1 / 2}(f)$ for broadband aLIGO and ET-D are shown for comparison. In each plot the numerical waveform monotonically approaches the EOB waveform as the tidal parameter $\Lambda$ decreases. Matching and splicing conventions are those of Fig. 2.

waveform fixed because we must specify a matching window $T_{I}<t<T_{F}$, and as discussed below, there is only a small region of the numerical waveforms over which a valid match can be performed. Once the values of $\tau$ and $\Phi$ are determined, we will then choose to instead hold the EOB waveform fixed and shift the numerical waveform in the opposite direction by rewriting it as $h_{\mathrm{NR}}^{\text {shift }}(t)=h_{\mathrm{NR}}(t+\tau) e^{+i \Phi}$. This is done so that all of the numerical waveforms with the same $Q$ and $M_{\mathrm{NS}}$ are aligned relative to a single fixed reference EOB waveform.

Over a matching window $T_{I}<t<T_{F}$ (bounded by solid vertical lines in Fig. 2), the normalized match between the waveforms is defined as

$$
m(\tau, \Phi)=\frac{\operatorname{Re}\left[z(\tau) e^{i \Phi}\right]}{\sigma_{\mathrm{NR}} \sigma_{\mathrm{EOB}}(\tau)}
$$


where

$$
z(\tau)=\int_{T_{I}}^{T_{F}} h_{\mathrm{NR}}(t) h_{\mathrm{EOB}}^{*}(t-\tau) d t
$$

and the normalizations for each waveform in the denomenator are defined as

$$
\sigma_{\mathrm{NR}}^{2}=\int_{T_{I}}^{T_{F}}\left|h_{\mathrm{NR}}(t)\right|^{2} d t
$$

and

$$
\sigma_{\mathrm{EOB}}^{2}(\tau)=\int_{T_{I}}^{T_{F}}\left|h_{\mathrm{EOB}}(t-\tau)\right|^{2} d t .
$$

The time-shift $\tau$ and phase $\Phi$ are chosen to maximize the match $m(\tau, \Phi)$ for a fixed matching window. Explicitly, the phase is determined analytically to be $\Phi=-\arg [z(\tau)]$; plugging this result back into Eq. (4), the time-shift is given by maximizing $|z(\tau)| /\left[\sigma_{\mathrm{NR}} \sigma_{\mathrm{EOB}}(\tau)\right]$ over $\tau$. As stated above, once $\tau$ and $\Phi$ are found we shift the numerical waveform in the opposite direction to generate $h_{\mathrm{NR}}^{\text {shift }}(t)=h_{\mathrm{NR}}(t+\tau) e^{+i \Phi}$.

A hybrid waveform is generated by smoothly turning off the EOB waveform and smoothly turning on the shifted numerical waveform over a splicing window $S_{I}<t<S_{F}$ (bounded by dotted vertical lines in Fig. 2) which can be chosen independently of the matching window. As in Ref. [13], we employ Hann windows

$$
\begin{aligned}
& w_{\text {off }}(t)=\frac{1}{2}\left[1+\cos \left(\frac{\pi\left[t-S_{I}\right]}{S_{F}-S_{I}}\right)\right] \\
& w_{\text {on }}(t)=\frac{1}{2}\left[1-\cos \left(\frac{\pi\left[t-S_{I}\right]}{S_{F}-S_{I}}\right)\right] .
\end{aligned}
$$

The hybrid waveform is then written

$$
h_{\text {hybrid }}(t)=\left\{\begin{array}{lc}
h_{\mathrm{EOB}}(t) & t<S_{I} \\
w_{\mathrm{off}}(t) h_{\mathrm{EOB}}(t)+w_{\mathrm{on}}(t) h_{\mathrm{NR}}^{\mathrm{shift}}(t) & S_{I}<t<S_{F} \\
h_{\mathrm{NR}}^{\text {shift }}(t) & t>S_{F}
\end{array} .\right.
$$

As shown in Fig. 2, we choose the start of the splicing interval to be the same as the start of the matching window $S_{I}=T_{I}$ and choose the end of the splicing window to be $S_{F}=T_{I}+4 \mathrm{~ms}$. It is also necessary to use these windows to smoothly turn on the hybrid waveform at low frequency when performing a discrete Fourier transform to avoid the Gibbs phenomenon. Unlike the case for BNS waveforms, it is not necessary to window the end of the hybrid waveform as the amplitude rapidly decays to zero anyway during the ringdown.

\section{Parameter estimation}

The output of a gravitational-wave detector $s(t)=n(t)+h(t)$ is the sum of detector noise $n(t)$ and a possible gravitational-wave signal $h(t)$. Stationary, Gaussian noise is characterized by its power spectral density (PSD) $S_{n}(|f|)$ defined by

$$
\left\langle\tilde{n}(f) \tilde{n}^{*}\left(f^{\prime}\right)\right\rangle=\frac{1}{2} \delta\left(f-f^{\prime}\right) S_{n}(|f|)
$$

The gravitational wave signal is given in terms of the two polarizations of the gravitational wave by

$$
h(t)=F_{+} h_{+}(t)+F_{\times} h_{\times}(t),
$$

where $F_{+, \times}$are the detector response functions and depend on the location of the binary and the polarization angle of the waves. We assume the binary is optimally located at the zenith of the detector and optimally oriented with its orbital plane parallel to that of the detector. This condition is equivalent to averaging $h_{+}$and $h_{\times}$ $\left(F_{+}=F_{\times}=1 / 2\right)$. 
It is well known [18] that the optimal statistic for detection of a known signal $h(t)$ in additive Gaussian noise is

$$
\rho=\frac{(h \mid s)}{\sqrt{(h \mid h)}}
$$

where the inner product between two signals $h_{1}$ and $h_{2}$ is given by

$$
\left(h_{1} \mid h_{2}\right)=4 \operatorname{Re} \int_{0}^{\infty} \frac{\tilde{h}_{1}(f) \tilde{h}_{2}^{*}(f)}{S_{n}(f)} d f .
$$

In searches for gravitational-wave signals from compact binary mergers, a parametrized signal $h\left(t ; \theta^{A}\right)$ is known in advance of detection, and the parameters $\theta^{A}$ must be estimated from the measured detector output $s(t)$. The parameters $\theta^{A}$ of an inspiral are estimated by maximizing the inner product of the signal $s(t)$ over the template waveforms $h\left(t ; \theta^{A}\right)$. In the high signal-to-noise limit, the statistical uncertainty in the estimated parameters $\hat{\theta}^{A}$ arising from the instrumental noise can be estimated using the Fisher matrix

$$
\Gamma_{A B}=\left.\left(\frac{\partial h}{\partial \theta^{A}} \mid \frac{\partial h}{\partial \theta^{B}}\right)\right|_{\hat{\theta}^{A}} .
$$

Note that $\hat{\theta}^{A}$ are the parameter values that maximize the signal-to-noise. The variance $\sigma_{A}^{2}=\sigma_{A A}=\left\langle\left(\Delta \theta^{A}\right)^{2}\right\rangle$ and covariance $\sigma_{A B}=\left\langle\Delta \theta^{A} \Delta \theta^{B}\right\rangle$ of the parameters are then given in terms of the Fisher matrix by

$$
\left\langle\Delta \theta^{A} \Delta \theta^{B}\right\rangle=\left(\Gamma^{-1}\right)^{A B}
$$

For hybrid waveforms, the partial derivatives in the Fisher matrix must be approximated with finite differences. It is most robust to compute the derivatives of the Fourier transforms used in the inner product. We rewrite the Fourier transform of each waveform in terms of the amplitude $A$ and phase $\Phi$ as $\exp [\ln A-i \Phi]$. The derivatives $\partial \ln A / \partial \theta^{A}$ and $\partial \Phi / \partial \theta^{A}$ are then evaluated with finite differencing.

In general, errors in the parameters $\theta^{A}$ are correlated with each other forming an error ellipsoid in parameter space determined by the Fisher matrix $\Gamma_{A B}$. The uncorrelated parameters that are best extracted from the signal are found by diagonalizing $\Gamma_{A B}$. These new parameters are linear combinations of the original parameters $\theta^{A}$. We focus attention below on the two parameters $\log \left(p_{1}\right)$ and $\Gamma$, and fix all other parameters. We therefore construct the error ellipses in $\left\{\log \left(p_{1}\right), \Gamma\right\}$ parameter space and identify the parameter with the smallest statistical errors.

For the BHNS systems discussed here, the greatest departure from BBH behavior occurs for gravitationalwave frequencies in the range 500-5000 Hz. As a result, detector configurations optimized for detection of BHNS systems with low noise in the region below $500 \mathrm{~Hz}$ are not ideal for estimating EOS parameters. We therefore present results for the broadband aLIGO noise curve [19] and the ET-D noise curve [20] shown in Fig. 4.

In Fig. 5, we show the resulting 1- $\sigma$ error ellipses in the 2-dimensional parameter space $\left\{\log \left(p_{1}\right), \Gamma\right\}$ for an optimally oriented BHNS with $Q=2$ and $M_{\mathrm{NS}}=1.35 M_{\odot}$ at a distance of $100 \mathrm{Mpc}$. Surfaces of constant $\Lambda^{1 / 5}$ and NS radius, which are almost parallel to each other, are also shown. One can see that the error ellipses are aligned with these surfaces. This indicates that, as expected, $\Lambda^{1 / 5}$ is the parameter that is best extracted from BHNS gravitational-wave observations. Because $\Lambda^{1 / 5}$ and $R$ are so closely aligned we will use these two parameters interchangeably.

As mentioned above, there is some freedom in construction of the hybrid waveforms. The size and orientation of the error ellipses also depend on the details of this construction. We find that as long as the matching window is longer than approximately four gravitational-wave cycles to average out the effects of eccentricity and does not include the first two gravitational-wave cycles, the orientation of the error ellipses does not change significantly. As expected, the size of the ellipses decreases as more of the numerical waveform is incorporated into the hybrid 


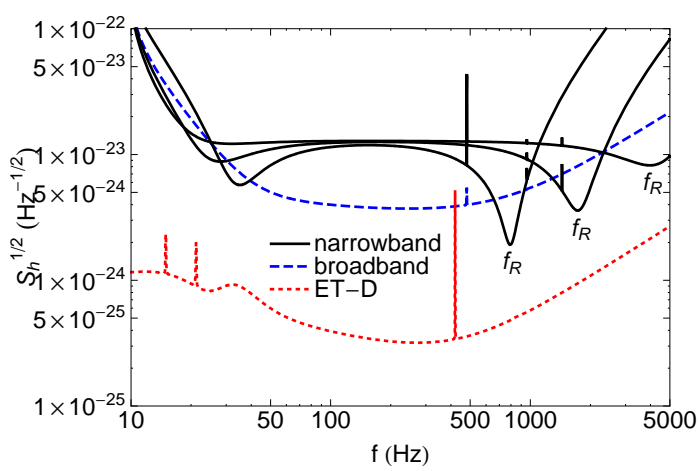

FIG. 4: Noise PSD for broadband aLIGO (dashed blue), ET-D (dotted red) and various configurations of narrowband aLIGO (solid black). The minima of the narrowband configuration are labeled $f_{R}$
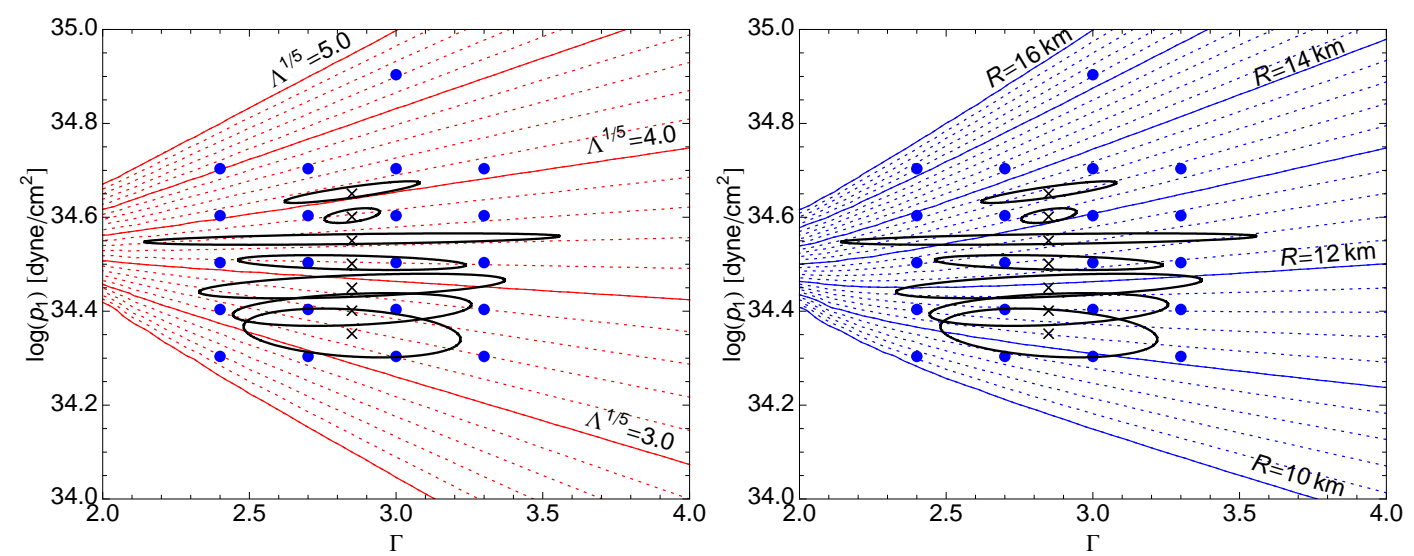

FIG. 5: $1-\sigma$ error ellipses for ET-D. Evenly spaced contours of constant $\Lambda^{1 / 5}(R)$ are also shown on the left (right). Matching and splicing conventions are those of Fig. 2.

waveform. We therefore adopt the last $12 \mathrm{~ms}$ before merger of each numerical waveform as the matching window and the first $4 \mathrm{~ms}$ of the matching window for splicing as shown in Fig. 2.

We have emphasized that, to within present numerical accuracy, the late-inspiral waveform is determined by the single parameter $\Lambda^{1 / 5}$. This implies that, by using countours of constant $\Lambda$ in the EOS space, one could have obtained the constraint on the EOS, summarized in Fig. 5 by varying only a single EOS parameter. For the simulations with other mass ratios and neutron star masses, we have used as our single parameter $\log \left(p_{1}\right)$ and not $\Gamma$ because countours of constant $p_{1}$ more closely coincide with contours of constant $\Lambda$ and because $\Lambda$ is a one to one function of $\log \left(p_{1}\right)$ throughout the parameter space. The one-parameter Fisher matrix can then be evaluated with finite differencing using the waveforms and values of $\Lambda$ at two points in EOS parameter space with different $\log \left(p_{1}\right)$.

The uncertainties in $\Lambda^{1 / 5}$ and $R$ are shown in Fig. 6 for broadband aLIGO. The uncertainty in these quantities is $\sim 10-40 \%$ for broadband aLIGO and are shifted down by an order of magnitude to $\sim 1-4 \%$ for ET-D. The uncertainties for the higher mass ratio $Q=3$ are somewhat larger than for $Q=2$, but not significantly so. It is not clear how rapidly the uncertainty in $\Lambda^{1 / 5}$ and $R$ will increase as the mass ratio is increased toward more realistic values. On the one hand the tidal distortion is likely to be much smaller for larger $Q$. On the other hand the overall signal will be louder, and the merger and ringdown will occur at lower frequencies where the noise is lower. Additional simulations for higher $Q$ are needed to address this question. 

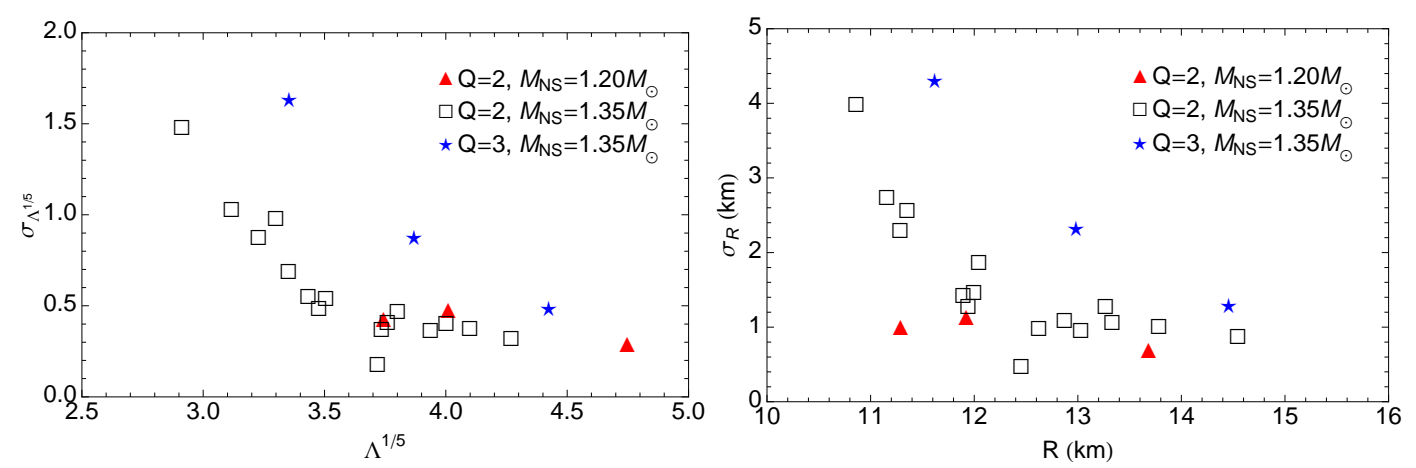

FIG. 6: $1-\sigma$ uncertainty $\sigma_{\Lambda^{1 / 5}}$ and $\sigma_{R}$ as a function of the parameters $\Lambda^{1 / 5}$ or $R$ for the broadband aLIGO noise PSD. Matching and splicing conventions are those of Fig. 2.

The presence of a signal-recycling cavity in the aLIGO instruments will allow them to be tuned to have improved narrowband sensitivity at the expense of bandwith. We have examined several narrowband tunings with central frequencies that vary between approximately $f_{R}=500 \mathrm{~Hz}$ and $4000 \mathrm{~Hz}$. Three of these noise curves are shown in Fig. 4. In Fig. 7 we plot the $1-\sigma$ uncertainty in NS radius $\sigma_{R}$ as a function of the narrowband central frequency $f_{R}$. For the waveforms considered in this paper the optimal narrowband frequency is in the range $1000 \mathrm{~Hz} \lesssim f_{R} \lesssim 2500 \mathrm{~Hz}$ and depends on the mass ratio, NS mass, and EOS. Narrowband configurations usually give smaller errors than the broadband configuration if $f_{R}$ happens to be tuned to within a few hundred $\mathrm{Hz}$ of the minimum for that BHNS event.
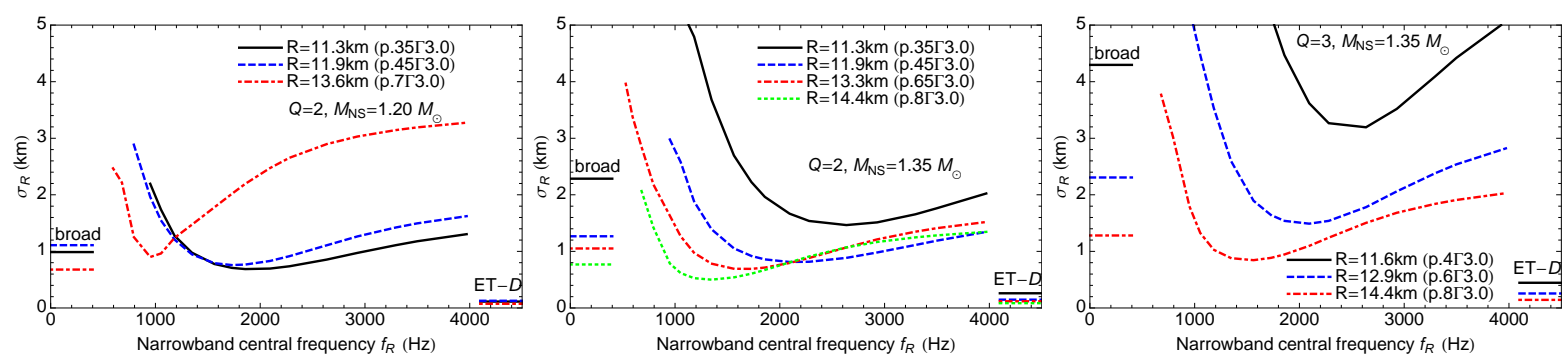

FIG. 7: $1-\sigma$ uncertainty in $R$ for different configurations of narrowband aLIGO and for different EOS. $f_{R}$ defines the frequency where $S_{n}$ is a minimum as shown in Fig. 4. Horizontal lines on the left and right indicate the corresponding $1-\sigma$ errors for broadband aLIGO and ET-D respectively. Matching and splicing conventions are those of Fig. 2.

\section{Discussion}

Using a large set of simulations incorporating a two-parameter EOS, we have found that the tidal deformability $\Lambda^{1 / 5}$, or equivalently the NS radius $R$, is the parameter that will be best extracted from BHNS waveforms. These parameters can be estimated to $10-40 \%$ with broadband aLIGO for an optimally oriented BHNS binary at $100 \mathrm{Mpc}$. The narrowband aLIGO configuration can do slightly better if it is tuned to within a few hundred $\mathrm{Hz}$ of the ideal frequency for a given BHNS event. The proposed Einstein Telescope will have an order-of-magnitude better sensitivity to the EOS parameters.

The results presented here can be compared with recent work to determine the mass and radius of individual NS in Type-1 X-ray bursts. Özel et al. [21] have obtained mass and radius measurements from several systems by simultaneously measuring the flux $F$, which is likely close to the Eddington value, and the blackbody temperature $T$ during X-ray bursts of systems with accurately determined distances. They obtain estimates of NS mass and 
radii with $\mathcal{O}(10 \%) 1-\sigma$ uncertainty. These radius error estimates are slightly smaller than those for the BHNS systems we have considered at $100 \mathrm{Mpc}$. However, we note that binary inspiral observations are subject to less systematic uncertainty due to questions of composition of the photosphere and associating it with the NS surface.

The uncertainty in NS radius for the merger and ringdown of BHNS systems examined here is of roughly the same size as that found for the last few orbits up to merger of BNS systems at the same $100 \mathrm{Mpc}$ distance $[13,22]$. BNS inspirals, however, will likely occur more frequently, and, including a tidally corrected inspiral-numerical hybrid, BNS systems are likely to have uncertainties that are smaller than BHNS systems by a factor of a few. Considering the post-merger phase for BNS waveforms may also provide additional information. Expected NS masses in both BNS and BHNS systems are slightly smaller than those measured for X-ray bursters which have accreted matter from their companion, so BNS and BHNS GW observations may complement X-ray burst observations by better constraining the lower density range of the EOS which is not well constained from X-ray burst observations $[21,23]$.

[1] J. Abadie, B. P. Abbott, R. Abbott, M. Abernathy, T. Accadia, F. Acernese, C. Adams, R. Adhikari, P. Ajith, B. Allen, et al., Classical and Quantum Gravity 27, 173001 (2010), 1003.2480.

[2] K. Belczynski, M. Dominik, T. Bulik, R. O’Shaughnessy, C. Fryer, and D. E. Holz, 715, L138 (2010), 1004.0386.

[3] K. Belczynski, V. Kalogera, and T. Bulik, Astrophys. J. 572, 407 (2002), arXiv:astro-ph/0111452.

[4] T. Hinderer, Astrophys. J. 677, 1216 (2008), 0711.2420.

[5] É. É. Flanagan and T. Hinderer, Phys. Rev. D 77, 021502 (2008), 0709.1915.

[6] J. Vines and É. É. Flanagan, ArXiv e-prints (2010), 1009.4919.

[7] J. Vines, T. Hinderer, and É. É. Flanagan, ArXiv e-prints (2011), 1101.1673.

[8] T. Damour and A. Nagar, Phys. Rev. D 80, 084035 (2009), 0906.0096.

[9] T. Binnington and E. Poisson, Phys. Rev. D 80, 084018 (2009), 0906.1366.

[10] M. Vallisneri, Physical Review Letters 84, 3519 (2000), arXiv:gr-qc/9912026.

[11] J. S. Read, B. D. Lackey, B. J. Owen, and J. L. Friedman, Phys. Rev. D 79, 124032 (2009), 0812.2163.

[12] J. M. Lattimer and M. Prakash, Astrophys. J. 550, 426 (2001), arXiv:astro-ph/0002232.

[13] J. S. Read, C. Markakis, M. Shibata, K. Uryū, J. D. E. Creighton, and J. L. Friedman, Phys. Rev. D 79, 124033 (2009), 0901.3258.

[14] K. Kyutoku, M. Shibata, and K. Taniguchi, Phys. Rev. D 82, 044049 (2010), 1008.1460.

[15] K. Kyutoku, H. Okawa, M. Shibata, and K. Taniguchi, ArXiv e-prints (2011), 1108.1189.

[16] P. B. Demorest, T. Pennucci, S. M. Ransom, M. S. E. Roberts, and J. W. T. Hessels, Nature (London) 467, 1081 (2010), 1010.5788 .

[17] T. Damour and A. Nagar, ArXiv e-prints (2009), 0906.1769.

[18] L. A. Wainstein and V. D. Zubakov, Extraction of signals from noise (Prentice-Hall, Englewood Cliffs, NJ, 1962).

[19] D. Shoemaker (LSC, 2009), URL https://dcc.ligo.org/cgi-bin/DocDB/ShowDocument?docid= 2974.

[20] S. Hild, M. Abernathy, F. Acernese, P. Amaro-Seoane, N. Andersson, K. Arun, F. Barone, B. Barr, M. Barsuglia, M. Beker, et al., Classical and Quantum Gravity 28, 094013 (2011), 1012.0908.

[21] F. Özel, G. Baym, and T. Güver, Phys. Rev. D 82, 101301 (2010), 1002.3153.

[22] L. Baiotti, J. Creighton, B. Giacomazzo, K. Kyutoku, C. Markakis, J. Read, L. Rezzolla, M. Shibata, K. Taniguchi, and J. Friedman, in progress (2011).

[23] F. Özel and D. Psaltis, Phys. Rev. D 80, 103003 (2009).

[24] The tidal deformability for the $\ell$ th multipole is often defined in terms of the NS radius $R$ and its dimensionless $\ell$ th Love number $k_{\ell}$ by $\lambda_{\ell}=\frac{2}{(2 \ell-1) ! ! G} k_{\ell} R^{2 \ell+1}$. Here we will discuss only the $\ell=2$ term so we write $\lambda:=\lambda_{2}$. 